\title{
The Role of a Nascent Polypeptide-Associated Complex Subunit Alpha in Siderophore Biosynthesis, Oxidative Stress Response, and Virulence in Alternaria alternata
}

\author{
Pin-Hua Wang, ${ }^{1}$ Pei-Ching Wu, ${ }^{1}$ Richie Huang, ${ }^{1}$ and Kuang-Ren Chung ${ }^{1,2,+}$ \\ ${ }^{1}$ Department of Plant Pathology, College of Agriculture and Natural Resources, National Chung Hsing University, Taichung, \\ 40227, Taiwan \\ ${ }^{2}$ Ph.D. Program in Microbial Genomics, National Chung Hsing University and Academia Sinica, Taichung 40227, Taiwan
}

Accepted 13 January 2020.

\begin{abstract}
The present study demonstrates that a nascent polypeptideassociated complex $\alpha$ subunit (Nac1) functions as a transcriptional regulator and plays both positive and negative roles in a vast array of functions in Alternaria alternata. Gain- and lossof-function studies reveal that Nac1 is required for the formation and germination of conidia, likely via the regulation of Fus3 and Sit2 mitogen-activated protein kinase (MAPK)coding genes, both implicated in conidiation. Nac1 negatively regulates hyphal branching and the production of cell walldegrading enzymes. Importantly, Nac1 is required for the biosynthesis of siderophores, a novel phenotype that has not been reported to be associated with a Nac in fungi. The expression of Nac1 is positively regulated by iron, as well as by the Hog1 MAPK and the NADPH-dependent oxidase (Nox) complex. Nac1 confers cellular susceptibility to reactive oxygen species (ROS) likely via negatively regulating the expression of the genes encoding Yap1, Skn7, Hog1, and Nox, all involved in ROS resistance. The involvement of Nac1 in sensitivity to glucose-, mannitol-, or sorbitol-induced osmotic stress could be due to its ability to suppress the expression of $S k n 7$. The requirement of Nac1 in resistance to salts is unlikely mediated through the transcriptional activation of Hogl. Although Nac1 plays no role in toxin production, Nac1 is required for fungal full virulence. All observed deficiencies can be restored by reexpressing a functional copy of $\mathrm{NaCl}$, confirming that Nac1 contributes to the phenotypes. Thus, a dynamic regulation of gene expression via Nac1 is critical for developmental, physiological, and pathological processes of A. alternata.
\end{abstract}

Keywords: citrus, iron uptake, nascent peptide, ROS resistance, toxin, virulence

${ }^{\dagger}$ Corresponding author: K.-R. Chung; krchung@nchu.edu.tw

Funding: This research was supported by grants from the Ministry of Science and Technology (MOST) of Taiwan (grant numbers: MOST1052313-B-005-010-MY3, MOST106-2313-B-005-025-MY3, and MOST 1072923-B-005-003-MY3) to K.-R. Chung.

*The $\boldsymbol{e}$-Xtra logo stands for "electronic extra" and indicates that supplementary figures and a supplementary table are published online.

The author(s) declare no conflict of interest.

๑) 2020 The American Phytopathological Society
The pathogenic fungus Alternaria alternata can affect more than 100 plant species; many have host specificity because of the production of host-selective toxins (HSTs). To date, at least seven pathotypes of $A$. alternata, including tangerine, rough lemon, strawberry, apple, Japanese pear, and tomato, each of which produces a unique HST, have been recognized (Thomma 2003). The tangerine pathotype produces an Alternaria citri toxin (ACT), which induces rapid electrolyte leakage from citrus cells and is highly toxic to tangerine, grapefruit, and their hybrids (Kohmoto et al. 1993). In addition to HST, A. alternata must overcome the toxicity of reactive oxygen species (ROS) in order to colonize its host plant. Several key proteins or regulators involved in the ROS detoxification processes have been identified and characterized. Those include the Yap1 transcription regulator, the high osmolarity glycerol 1 (Hog1) mitogen-activated protein kinase (MAPK), the $\mathrm{Skn} 7$ response regulator, and the NADPH-dependent oxidase (Nox) complex (Chung 2012, 2014).

Recent studies have further revealed that siderophoremediated iron acquisition plays a critical role in ROS detoxification (Chen et al. 2014). A nonribosomal peptide synthetase (Nps6) has been demonstrated to be required for the biosynthesis of dimethyl coprogen siderophore and resistance to ROS in A. alternata (Chen et al. 2013). Fungal strains that fail to effectively detoxify toxic ROS are also severely defective in virulence. A low level of $\mathrm{H}_{2} \mathrm{O}_{2}$ produced by Nox likely functions as a signal cue, which could activate the expression of genes involved in ROS detoxification and resistance and siderophore biosynthesis. $\mathrm{H}_{2} \mathrm{O}_{2}$ produced by Nox could also promote the formation of a disulfide bond in Yap1, increase phosphorylation of Hog1, and trigger nuclear localization of Yap1 and Hog1 (Lin and Chung 2010; Lin et al. 2009). The expression of the Nps6 gene and the biosynthesis of dimethyl coprogen siderophore are regulated by Nox, Yap1, and Hog1 but not Skn7. Although $\mathrm{Skn} 7$ regulates iron uptake in A. alternata, $\mathrm{Skn} 7$ is not directly involved in siderophore production. $\mathrm{H}_{2} \mathrm{O}_{2}$ produced by Nox also plays a critical role in conidial formation (Yang and Chung 2012, 2013). Studies with A. alternata reveal that conidial formation is a complex process regulated by many pathways including the pheromone response Fus3 MAPK and the cell-wall integrity Slt2 MAPK-mediated signaling pathways (Lin et al. 2010; Yago et al. 2011).

The nascent polypeptide-associated complex (Nac) binds to ribosomes to ensure newly synthesized proteins are folded properly and to prevent an untimely interaction with other proteins in the cytosol (Pech et al. 2010; Preissler and Deuerling 2012; Rospert et al. 2002). Nac functions cooperatively with

668 / Molecular Plant-Microbe Interactions 
the heat shock protein chaperones (Hsp40/70) to release a newly synthesized peptide chain from ribosomes and deliver it to the appropriate subcellular compartment (Hartl and Hayer-Hartl 2002). Nac is a heterodimeric complex consisting of two subunits $(\alpha$ and $\beta$ ) and is very conserved in eukaryotes ranging from yeasts to humans. The budding yeast Saccharomyces cerevisiae has an additional subunit ( $\beta$ ') forming two different heterodimers $\alpha \beta$ and $\alpha \beta$ ' with distinct regulatory functions (del Alamo et al. 2011; Ott et al. 2015; Wang et al. 1995). S. cerevisiae mutants lacking Nac are temperature-sensitive and display defects in growth and cell morphology (George et al. 2002; Reimann et al. 1999). Nac has been thought to negatively regulate the translocation of proteins into the endoplasmic reticulum and positively regulate the translocation of proteins into the mitochondria (Rospert et al. 2002; Wiedmann and Prehn 1999).

In addition to the translational process, each of the Nac subunits can enter the nucleus and function as a transcriptional regulator (Franke et al. 2001). In the fission yeast Schizosaccharomyces pombe, Nac has been demonstrated to bind to a DNA junction (also known as a Holiday junction or $\mathrm{X}$ junction) (Whitby and Dixon 2001), which is an intermediate of the recombination processes and an important structure for DNA replication and gene expression. S. pombe mutants lacking Nac exhibit increased sensitivity to canavanine, an L-arginine analog, but are not temperature sensitive. While Nac is not essential in yeasts, mutations of Nac in Mus musculus, Drosophila melanogaster, and Caenorhabditis elegans are lethal, confirming the importance of Nac in in-vivo functions (Bloss et al. 2003; Deng and Behringer 1995; Markesich et al. 2000). Changes in intracellular levels of Nac have also been thought to be associated with several human diseases, including AIDS, Down syndrome, malignant brain tumors, and Alzheimer's disease (Kim et al. 2002; Kroes et al. 2000; Scheuring et al. 1998), as well as apoptosis and autophagy (Bloss et al. 2003; Creagh et al. 2009; Guo et al. 2014; Hotokezaka et al. 2009).

The biological and pathological roles of Nac in plantpathogenic fungi remain largely unknown. Studies with the plant-pathogenic fungus Sclerotinia sclerotiorum reveal the involvement of $\mathrm{Nac}$ in the formation of sclerotia ( $\mathrm{Li}$ et al. 2015). However, Nac plays a negative role in the regulation and production of cell wall-degrading enzymes (CWDEs) and full virulence, based on the analysis of fungal mutants generated from RNA interference (RNAi) in S. sclerotiorum. A Nac analog (formerly Ape1) of A. alternata, after being cloned, expressed in Escherichia coli, and applied onto cucumber leaves, induces plant defense reactions and reduces anthracnose incidence caused by Colletotrichum orbiculare (Lin et al. 2017). A Nac analog (PeaT1) of Alternaria tenuissima has been shown to induce salicylic acid-mediated systemic acquired resistance (SAR) in tobacco and confers resistance to tobacco mosaic virus (Zhang et al. 2011). Studies have identified several plant proteins that physically interact with a purified Nac analog and lead to abiotic stress tolerance or disease resistance (Meng et al. 2018; Shi et al. 2017).

To further understand the mechanisms underlying oxidative stress resistance and cellular responses to osmotic and oxidative stresses in A. alternata, the functions of the Nac1 homolog were determined by creating and analyzing loss- and gain-offunction mutants and a heterokaryotic strain. Overall, the results have revealed that Nac1 is required for the biosynthesis of dimethyl coprogen siderophores, resistance to sucrose and salt stress, conidial development, and virulence. Nac1 plays a negative role in the production of CWDEs and cellular resistance to ROS and glucose, mannitol, and sorbitol. The present study highlights the central regulatory role of Nac1 in developmental and biological functions and reveals the complexity of the molecular mechanisms involved in coordinating cellular response to various types of environmental stress in A. alternata.

\section{RESULTS}

\section{Characterization of the Nac1-coding gene.}

A. alternata Nacl was found to contain a 792-bp open reading frame interrupted by two small introns of 113 and $52 \mathrm{bp}$ that encodes a polypeptide of 208 amino acids. A Nac domain belonging to the NAC superfamily and a C-terminal ubiquitinassociated domain were found in Nac1. A conserved EGD2 domain belonging to NAC $\alpha$-basic transcriptional factor 3 (BTF3) was also found in Nac1. Sequence alignment and phylogenetic analysis revealed that $\mathrm{Nacl}$ is highly conserved among fungi. Analysis of $1.1 \mathrm{~kb}$ upstream of the putative ATG start codon of $\mathrm{NaCl}$ identified several putative binding sites for various transcriptional factors. The $5^{\prime}$ promoter region of Nacl was found to contain multiple GATA consensus motifs, potential binding regions for the light-regulated WC1/WC2 (Linden and Macino 1997) and the nitrogen-induced AreA (Marzluf 1997) transcriptional regulators. One ambient pH-regulated PacC-binding consensus motif (GCCARG) (Espeso et al. 1997) and five cAMP-inducible C/EBPbinding motifs (CCAAT or CAAT) (Rangan et al. 1996) were found in the $5^{\prime}$ promoter region of $\mathrm{Nacl}$. The $\mathrm{Nacl}$ promoter was found to have three CATTCY consensus motifs, potential binding sites for the conidial formationrelated AbaA transcriptional activator described in Aspergillus nidulans (Adams et al. 1998).

\section{Identification of the A. alternata Nac1 mutants.}

The Nacl gene was mutated by inserting a hygromycin resistance gene $(H Y G)$ flanked by a promoter and a terminator of $\operatorname{TrpC}$ (Supplementary Fig. S1A). Transformation of two overlapping and truncated $H Y G$ fragments flanked with a $5^{\prime}$ or $3^{\prime}$ end of $\mathrm{NaCl}$ into protoplasts prepared from the wild-type strain of $A$. alternata resulted in identification of four fungal strains designated D1, D2, D7, and E10 from medium amended with hygromycin. D1, D2, and D7 strains ( $\Delta n a c 1)$ exhibited radial growth slower than wild-type and E10 strains. Southern blot analysis revealed that a $2.4-\mathrm{kb}$ band was identified from wildtype DNA digested with $\mathrm{XbaI}$ and hybridized to a $\mathrm{Nacl}$-specific probe (Supplementary Fig. S1B). A 1.2-kb band was identified from those of D1, D2, and D7 due to the presence of two additional $\mathrm{XbaI}$ sites, one within $H Y G$ and another in the $\operatorname{TrpC}$ terminus. Both 2.4- and 1.2-kb bands were identified in genomic DNA of E10, indicating that E10 was a heterokaryotic strain carrying both wild-type and mutated $\mathrm{Nacl}$ nuclei. E10 was obtained by consecutively streaking conidia three times for single colonies. Strains obtained after each streaking were examined by Southern blotting, resulting in similar hybridizing patterns (data not shown). Hybridization of genomic DNA to a $H Y G$-specific probe resulted in a single $2.1-\mathrm{kb}$ band in four transformants (Supplementary Fig. S1C). In addition to the 2.1$\mathrm{kb}$ band, an approximately 3-kb hybridizing band was identified by the $H Y G$ probe in E10. No bands were detected by the $H Y G$ probe in genomic DNA of wild type. Transformation of the pNac1::pCB1532 plasmid carrying a wild-type copy of Nacl into protoplasts prepared from the D1 mutant identified six transformants (complementation [Cp] strains $\mathrm{Cp} 1$ to $\mathrm{Cp} 6$ ) from medium amended with sulfonylurea. All six $\mathrm{Cp}$ strains had a full-length Nacl gene, based on PCR examination (data not shown).

\section{Nac1 is required for growth and hyphal branching.}

Fungal strains ( $\Delta$ nac1-D1, D2, and D7) carrying a defective Nacl locus reduced growth on potato dextrose agar (PDA) as 
much as $38 \%$ compared with wild type after a 5-day incubation (Fig. 1A and B). The E10 strain carrying heterokaryotic nuclei-some had a disrupted Nacl locus and others had a heterokaryotic $H Y G$ insertion-displayed a wild-type growth rate. Microscopic observation revealed that $\Delta n a c l$ hyphae were frequently branched (Fig. 1C and D). Quantitation of the number of hyphal branch revealed that $\Delta$ nacl increased hyphal branching by threefold compared with wild type and E10. The Cp strains displayed radial growth and hyphal branching similar to wild type (Supplementary Fig. S2).
A WT

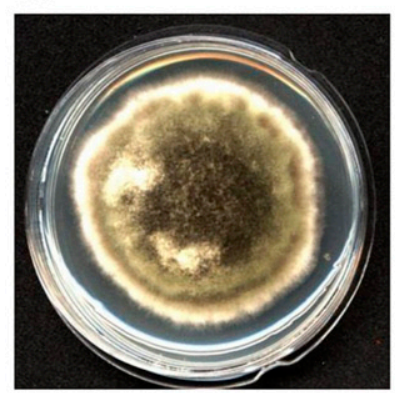

B
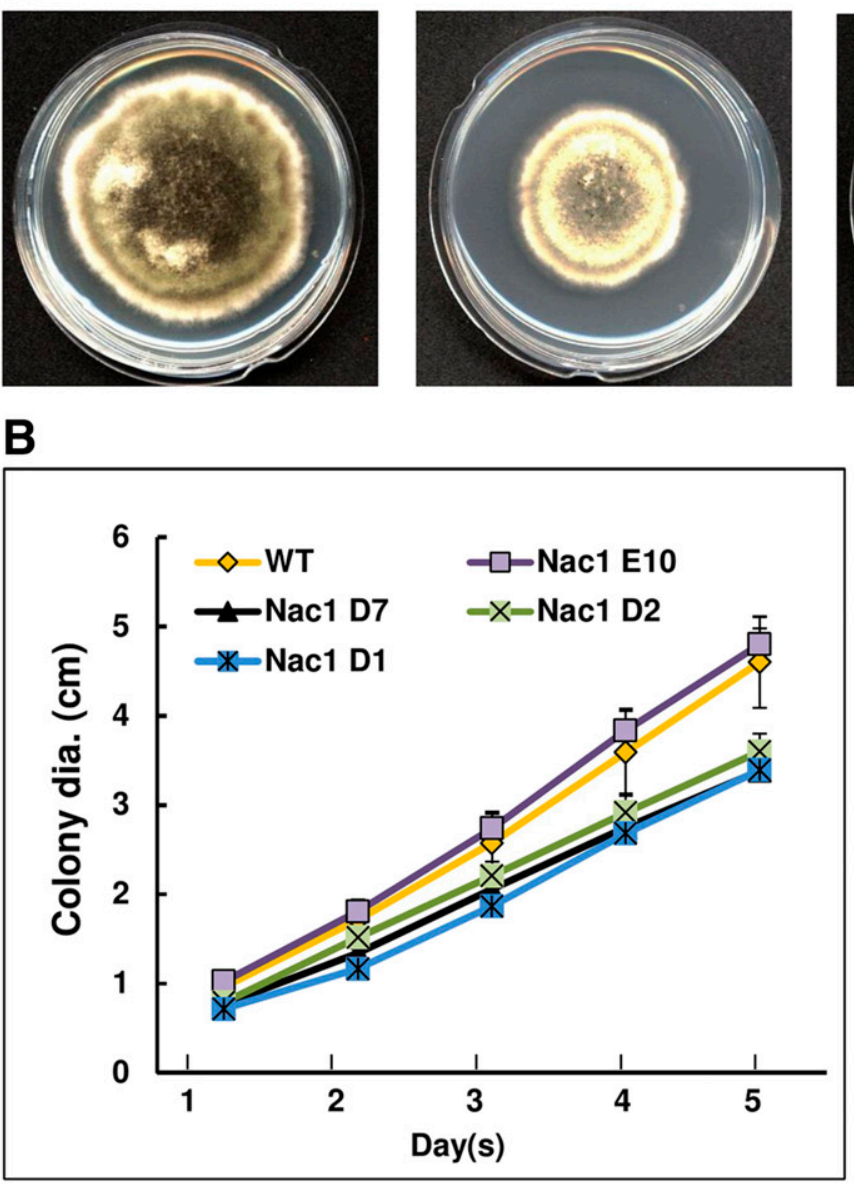

$\Delta$ nac1-D2

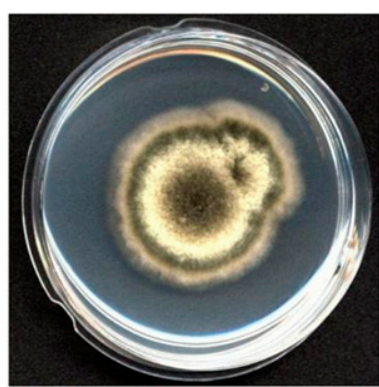

C

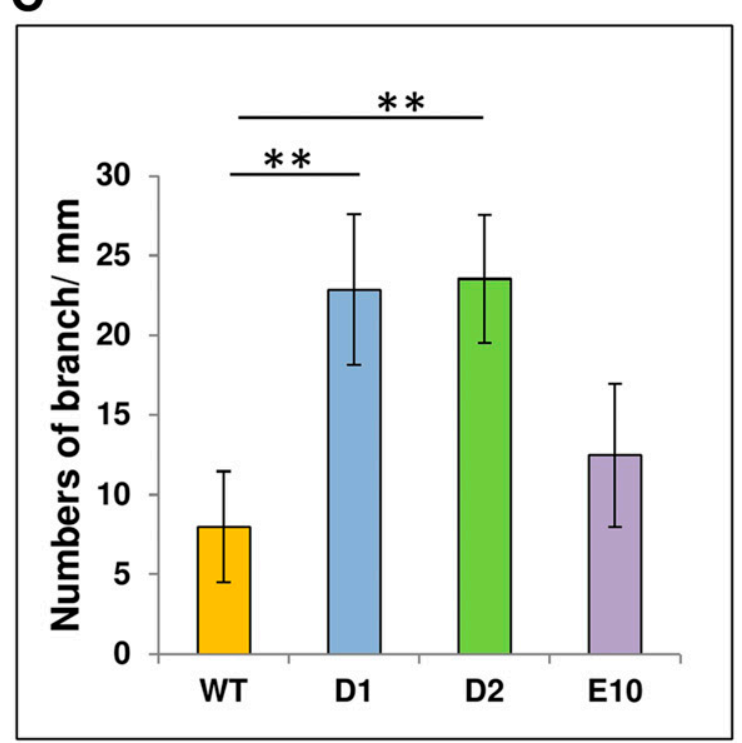

E10

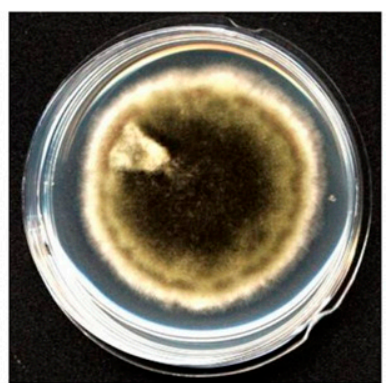

D

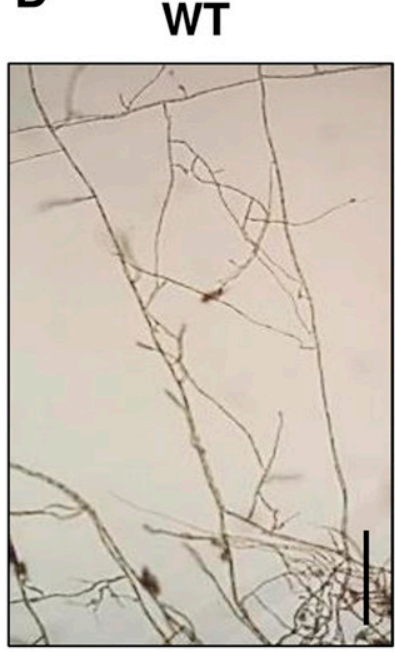

$\Delta$ nac1-D1

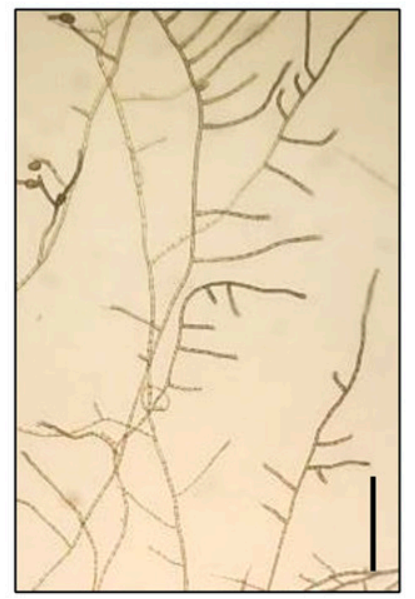

$\Delta$ nac1-D2

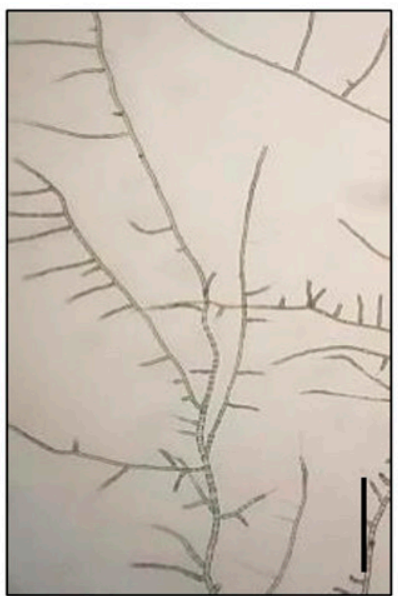




\section{Nac1 is required for the formation} and germination of conidia.

The wild-type and E10 strains produced abundant darkpigmented, ovate conidia with both cross and longitudinal septae (Fig. 2A). $\Delta$ nacl produced significantly fewer conidia than wild type and E10. Conidia produced by $\Delta$ nacl were much smaller than those produced by wild type (Fig. 2B). E10 produced conidia at quantities similar to wild type. However, the size of conidia produced by E10 was smaller than those of wild type and larger than those produced by $\Delta$ nacl (Fig. 2A and C). Conidia produced by $\Delta n a c 1$ and E10 germinated at rates slightly slower than those of wild type (Fig. 2D). The $\mathrm{Cp}$ strains produced conidia with sizes, quantities, and germination rates similar to wild type (Supplementary Fig. S2).
Nac1 is involved in the response to oxidative and osmotic stress.

$\Delta n a c 1$ reduced radial growth on PDA or PDA amended with dimethyl sulfoxide in relation to wild type and E10 by 36 to $38 \%$. Fungal strains were tested for sensitivity to various oxidants. On PDA amended with eosin $\mathrm{Y}(50 \mu \mathrm{M})$, rose bengal $(5 \mu \mathrm{M})$, 2-chloro-5-hydroxypyridine (CHP) (3 mM), or $\mathrm{H}_{2} \mathrm{O}_{2}$ $(5 \mathrm{mM}), \Delta$ nacl reduced growth by less than $20 \%$ in relation to wild type (Fig. 3A). When grown on minimal medium (MM) amended with rose bengal, $\mathrm{H}_{2} \mathrm{O}_{2}$, or tert-butyl-hydroperoxide, $\Delta$ nacl also reduced growth by less than $25 \%$ compared with wild type (Supplementary Fig. S3). The addition of hematoporphyrin, eosin Y, or CHP into MM had no effects on growth reduction of $\Delta n a c l$ compared with the control (medium only). The addition of glucose, mannitol, or sorbitol at $1.5 \mathrm{M}$ each into

A
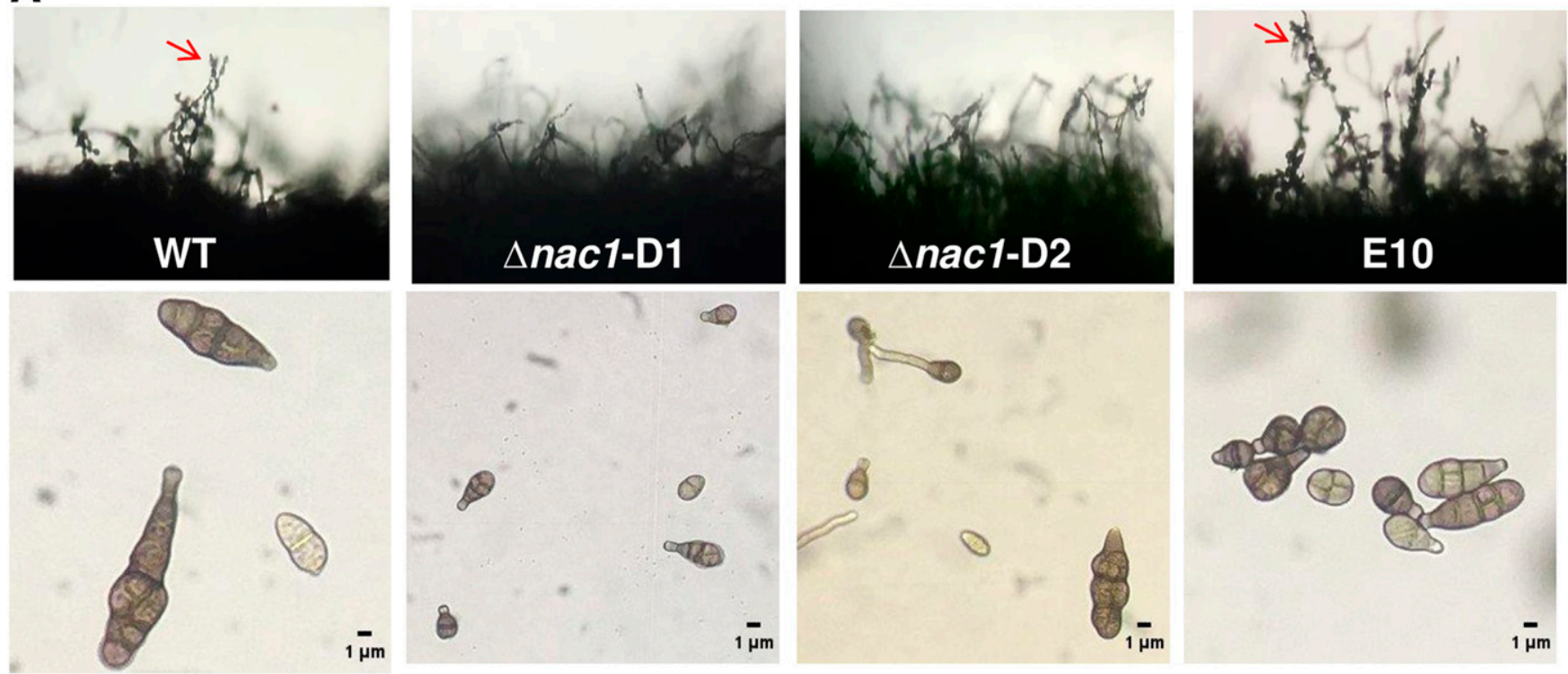

B

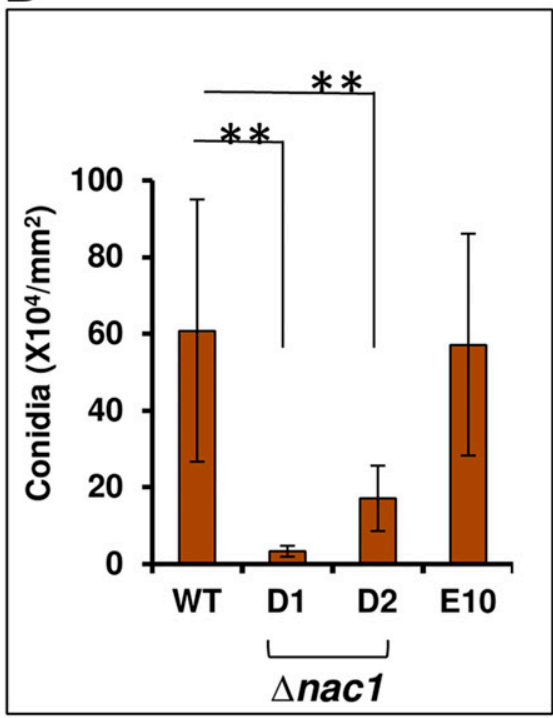

C

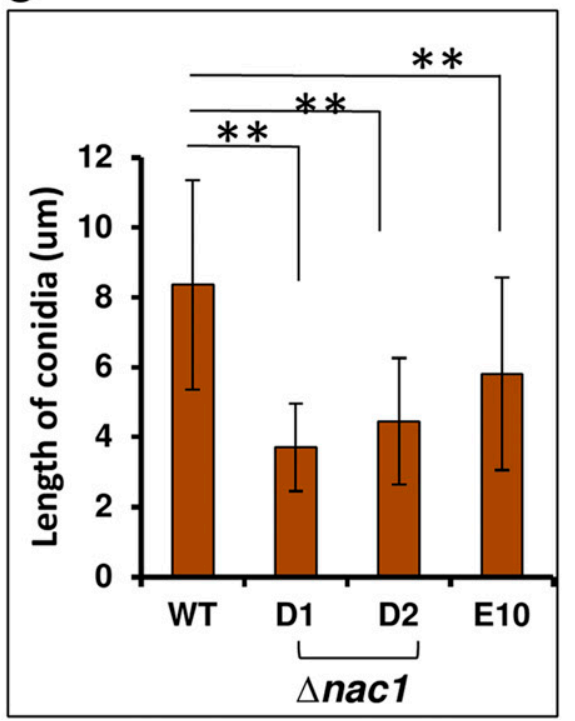

D

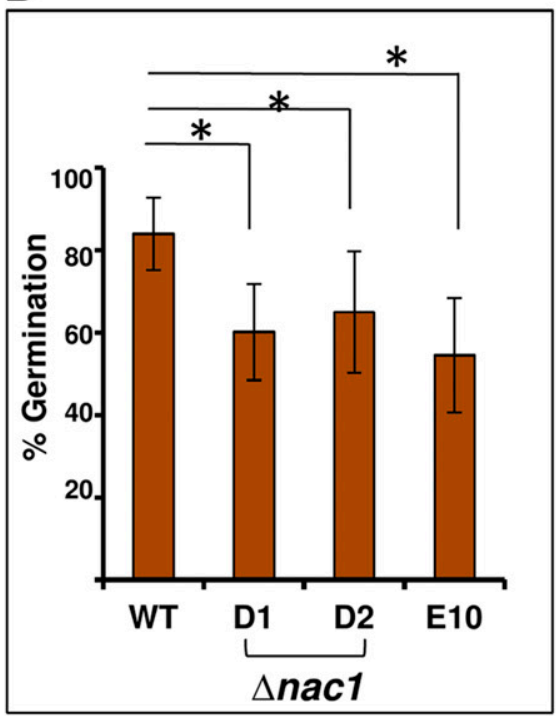

Fig. 2. The nascent polypeptide-associated complex $\alpha$ subunit (Nac1) is required for the formation, maturation, and germination of conidia. A, Conidia (indicated by arrows) produced by the wild type (WT), two Nac1 mutants (Anac1-D1 and $\Delta$ nac1-D2), and the E10 heterokaryotic strain of Alternaria alternata grown on potato dextrose agar after incubation for 3 days. The size of conidia produced by $\Delta n a c l$ is much smaller than those produced by WT and E10. B, Quantification of the number of conidia produced by A. alternata strains. C, Comparison of the lengths of conidia. D, The germination rate (\%) of conidia produced by $A$. alternata strains grown on glass slides for 6 h. Means indicated by asterisks are significantly different from one another, $P<0.05(*)$ and $P<$ 0.01 (**). 
PDA also resulted in less severe growth reduction (by 20 to $30 \%$ ) than those grown on PDA control (Fig. 3B). Anacl increased sensitivity to sucrose $(1.5 \mathrm{M}), \mathrm{NaCl}(1 \mathrm{M})$, and $\mathrm{KCl}$ (1 M) by displaying nearly $50 \%$ growth reduction compared with wild type. $\Delta$ nacl displayed wild-type sensitivity to canavanine (data not shown); the Cp strains displayed the wild-type sensitivity to all test chemicals (Supplementary Fig. S2).

\section{Nac1 is involved in siderophore production.}

Both the wild-type and E10 strains grown on a medium containing a chrome azurol S (CAS) dye and hexadecyltrimethyl ammonium bromide (HDTMA) resulted in distinct orange halos around colonies after 5 days of incubation (Fig. 4A and B). Iron, CAS, and HDTMA formed a blue-colored compound; removing iron by siderophores resulted in an orange-colored compound (Schwyn and Neilands, 1987). $\Delta$ nacl produced much smaller orange halos than those produced by wild type. Thin-layer chromatography (TLC) analysis
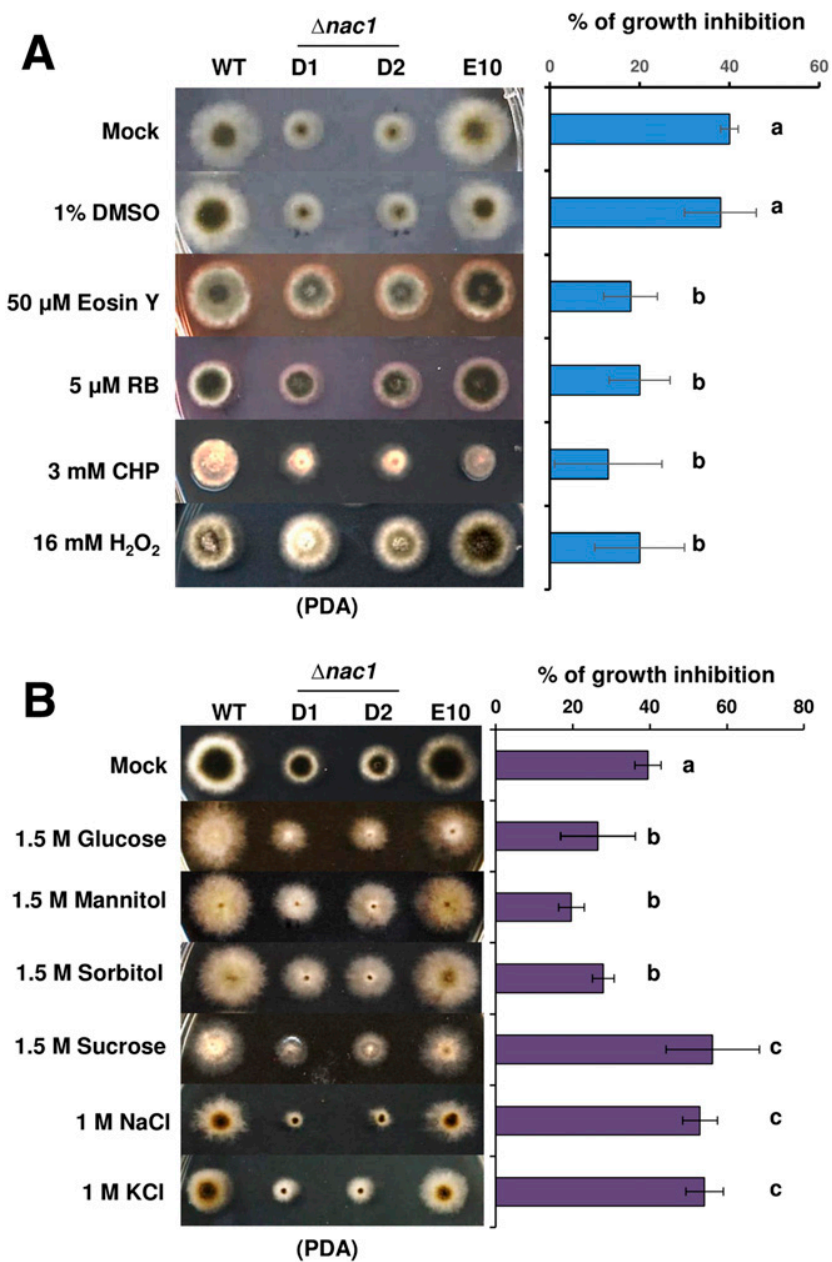

Fig. 3. The nascent polypeptide-associated complex $\alpha$ subunit (Nac1) plays an opposite role in oxidative and osmotic stress in Alternaria alternata. A, Images of the wild type (WT), two $\Delta$ nacl mutants (D1 and D2), and the E10 heterokaryotic strain grown on potato dextrose agar (PDA) amended with reactive oxygen species-generating chemicals (left panel). Quantitative analysis of chemical sensitivity (right panel). B, A. alternata strains grown on PDA amended with sugars or salts as indicated (left panel). Quantitative analysis of growth inhibition in the presence of osmotic stress (right panel). Growth inhibition was determined as a cumulative percentage of growth of WT and $\Delta$ nacl grown on the same plate. The data are the mean and standard deviation of two independent experiments with four biological replicates. Means indicated by the same letter are not significantly different from one another, $P<0.05$. of purified siderophores produced by wild type and E10 resulted in a reddish-orange band at $R_{f} 0.60$ to 0.62 (Fig. 4C). At similar fungal mass, siderophores purified from $\Delta n a c 1$ resulted in band intensities much fainter than those produced by wild type and E10. The expression of $\mathrm{Nacl}$ was assessed in the wildtype strain, by quantitative real-time PCR (qRT-PCR), following a shift of fungal hyphae from $\mathrm{MM}$ after 3 days of growth to MM containing $0.2 \mathrm{mM} \mathrm{FeCl}_{3}$ for $8 \mathrm{~h}$. The results revealed that exposure of wild type to iron increased the expression of $\mathrm{Nacl}$ over time (Fig. 4D), reaching a plateau at $4 \mathrm{~h}$. The Nacl transcript level was reduced after an 8-h incubation. The addition of the iron chelator bathophenanthrolinedisulfonic acid $(2 \mathrm{mM})$ suppressed the expression of $\mathrm{Nacl}$ (Fig. 4E), supporting further that the expression of $\mathrm{Nacl}$ was regulated by iron. The $\mathrm{Cp}$ strains displayed the wild-type siderophore production (Supplementary Fig. S2).

\section{Nac1 positively regulates the genes involved}

in the biosynthesis and transport of siderophores.

qRT-PCR was performed to assess the expression of the genes involved in the biosynthesis and transport of siderophores. Those included two genes required for siderophore biosynthesis, the Nps6 gene encoding a nonribosomal peptide synthetase and the SidA gene encoding an L-ornithine N5oxygenase, the two siderophore transporter-coding genes MirC
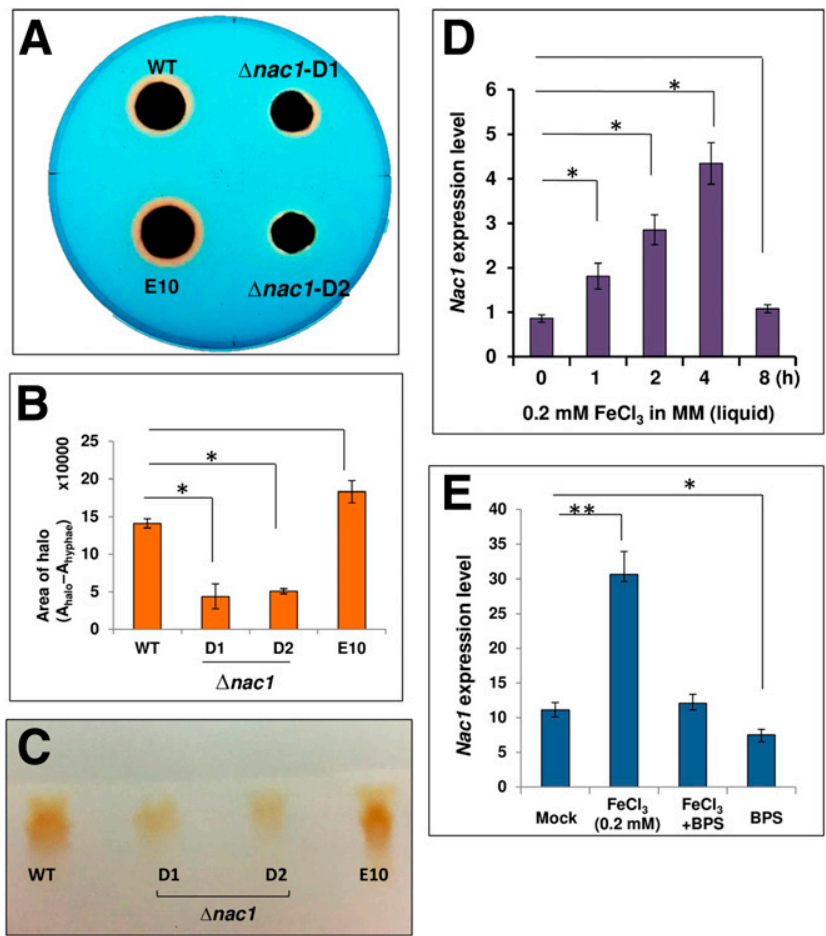

Fig. 4. The nascent polypeptide-associated complex $\alpha$ subunit (Nac1) is required for production of siderophores in Alternaria alternata. A, Production of siderophores (indicated by the formation of orange halos around colonies) by the wild type (WT), two $\Delta$ nac1 mutants (D1 and D2), and the E10 heterokaryotic strain grown on agar medium containing chrome azurol $\mathrm{S}$ for 5 days. Only a representative is shown. B, Quantification of areas of yellow halos (minus fungal colony) produced by $A$. alternata strains using an Image $\mathbf{J}$ program. C, Thin-layer chromatography analysis of siderophores purified from culture filtrates of fungal strains by passing through a column packed with Amberlite XAD-16 resins. Siderophores were separated with methanol/acetic acid/ $\mathrm{H}_{2} \mathrm{O}$ (4:1:5, vol/vol/vol). D, Real-time PCR analysis of the $\mathrm{Nacl}$ gene transcript in the WT strain grown on liquid minimal medium amended with $0.2 \mathrm{mM} \mathrm{FeCl}_{3}$ for varying times. $\mathbf{E}$, The addition of the iron chelator bathophenanthrolinedisulfonic acid (BPS, $2 \mathrm{mM}$ ) suppressed the expression of $\mathrm{Nacl}$. Means indicated by an asterisk are significantly different from one another, $P<0.05(*)$ and $P<0.01(* *)$. 
and Sit1, and the iron-responsive transcription factor-coding gene Atfl. The results revealed that the expression of all five genes was down-regulated markedly in the two $\Delta$ nacl strains compared with those of wild type (Fig. 5).

Nac1 plays a negative role in the production of CWDEs.

The overall activities of CWDEs produced by the Nacl mutants were evaluated and compared with wild type, E10, and the complementation strains. The Nacl mutants displayed increased activities of cellulase and pectinase compared with wild type and E10 (Fig. 6). The complementation strains displayed the wildtype production of CWDEs (Supplementary Fig. S2).

\section{Mutation of Nac1 affects fungal virulence.}

Fungal pathogenicity was assessed by inoculating conidial suspensions or hyphae on detached calamondin leaves. The results revealed that $\Delta n a c l$ barely induced necrotic lesions compared with wild type 3 days postinoculation (dpi) (Fig. 7A). The E10 strain induced necrotic lesions at rates and magnitudes similar to wild type (Fig. 7B). $\Delta n a c l$ was able to cause necrotic lesions indistinguishable from those induced by wild type and E10 3 dpi when citrus leaves were wounded prior to inoculation (Fig. 7C). The Cp strains displayed the wild-type virulence after inoculation in unwounded calamondin leaves (Fig. 7D). Bioassays of cell-free culture filtrates on calamondin leaves revealed that $\Delta n a c 1$ produced $\mathrm{ACT}$ at equal levels, comparable to those produced by wild type (Fig. 7E). TLC analysis also revealed no significant difference in the production of ACT between wild type and $\Delta$ nacl (Fig. 7F). Accumulation of $\mathrm{H}_{2} \mathrm{O}_{2}$ in citrus leaves in response to A. alternata was examined by 3,3'-diaminobenzidine (DAB) staining (Supplementary Fig. $\mathrm{S} 4$ ). Inoculation of wild type $12 \mathrm{~h}$ postinoculation resulted in the formation of a dark-brown pigment around the inoculation site, indicative of $\mathrm{H}_{2} \mathrm{O}_{2}$ accumulation. Inoculation of $\Delta n a c 1$ resulted in less $\mathrm{H}_{2} \mathrm{O}_{2}$ accumulation in citrus leaves.

\section{Nac1 plays both positive and negative roles in gene expression.}

The expression of the NoxA, NoxB, and NoxR genes encoding one of the NADPH oxidase subunits was determined by qRTPCR in wild-type and $\Delta n a c l$ strains. The results revealed that all three Nox genes were up-regulated in the $\Delta n a c l$ strain compared with those of wild type (Fig. 8). Deletion of $\mathrm{NaCl}$ also resulted in an increased expression of the gene encoding the stress-response regulators Skn7 and Yap1 as well the high osmolarity glycerol 1 (Hog1) MAPK. However, the expression of the genes encoding the cell-wall integrity Slt2 MAPK and the pheromone response Fus3 MAPK was downregulated considerably in two $\Delta$ nacl strains. The expression of Nacl was downregulated in fungal strains lacking NoxA, NoxB, NoxR, or Hogl. The expression of $\mathrm{Nacl}$ was not affected in fungal strains lacking $S k n 7$ or Yapl.

\section{DISCUSSION}

The Nac is a conserved protein involved in the proper folding of newly synthesized proteins in eukaryotes (Pech et al. 2010;
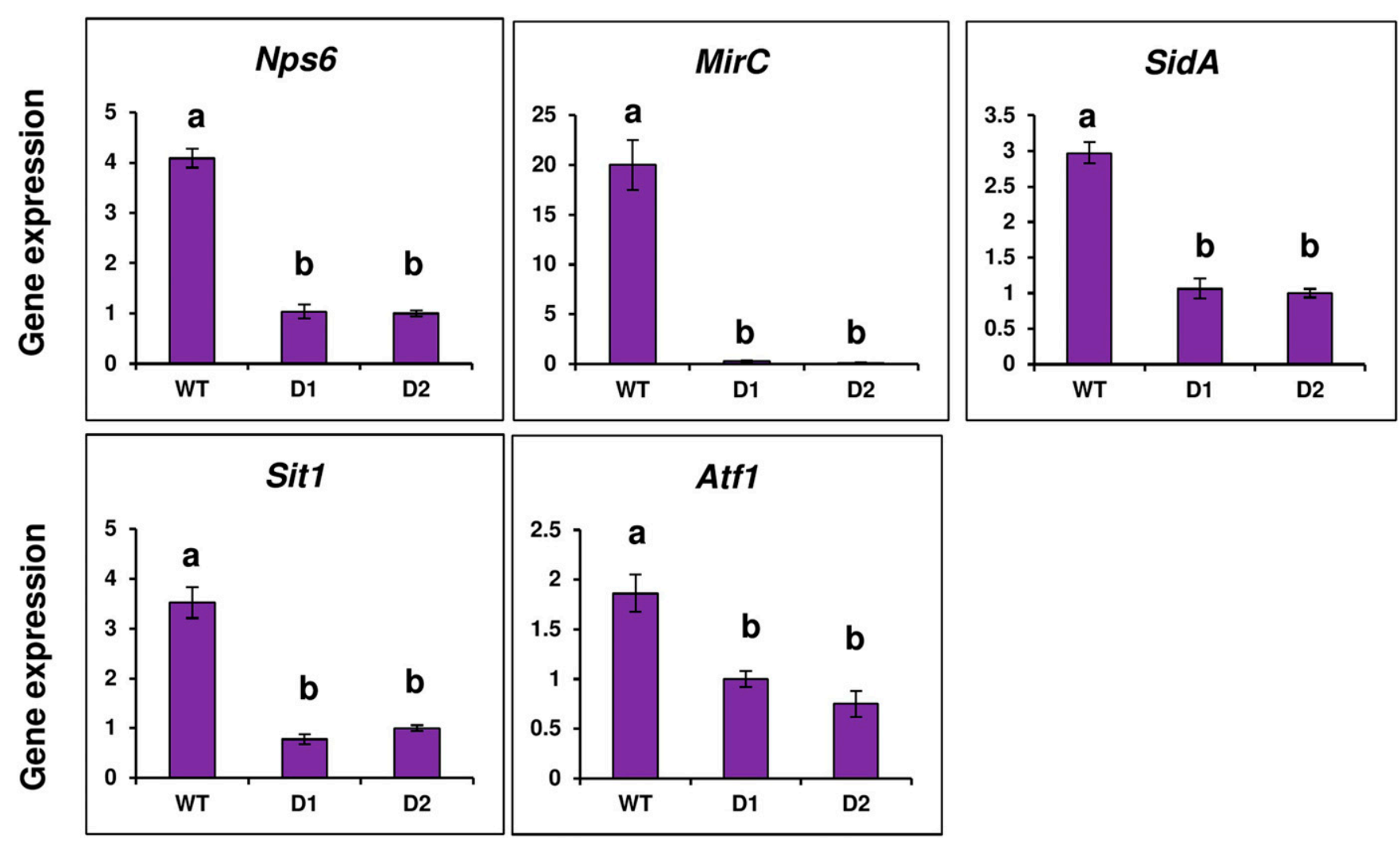

Fig. 5. The nascent polypeptide-associated complex $\alpha$ subunit (Nac1) is required for the expression of the genes involved in biosynthesis, regulation, and transportation of siderophores in Alternaria alternata. Quantitative real-time PCR analysis of the expression of the Nps6 gene encoding a nonribosomal peptide synthetase, the SidA gene encoding an L-ornithine N5-oxygenase, two siderophore transporter-coding genes, namely, MirC and Sit1, and an iron-responsive transcription factor-coding gene, Atfl, in the wild type (WT) strain, and two $\Delta$ nacl mutants (D1 and D2). Fungal strains were grown in potato dextrose broth (PDB) for 3 days and were subjected to RNA purification. RNA was treated with DNase and was used for the synthesis of the first-strand complementary DNA (cDNA), using an oligo-dT primer. cDNA was used as a template to amplify each of the gene fragments with gene-specific primers. The relative expression levels from three independent reactions were calculated using a comparative cycle threshold method in relation to the expression of the fungal $\beta$ tubulin-coding gene. Means indicated by the same letter are not significantly different from one another, $P<0.05$. All experiments were repeated at least two times, showing similar trends. 
Preissler and Deuerling 2012; Rospert et al. 2002). The Nac subunits can also independently enter the nucleus and act as a transcriptional regulator (Franke et al. 2001). In the present study, the biological function of the Nacl gene encoding a Nac subunit alpha was determined in the tangerine pathotype of A. alternata. The results indicate that $\mathrm{Nac} 1$ plays both positive and negative roles in a variety of biochemical and pathological activities via transcriptional regulation. Inserting a $H Y G$ cassette within the $\mathrm{Nacl}$ gene yielded mutants that display pleiotropic phenotypes. All observed phenotypes can be restored by reintroducing and expressing a functional copy of $\mathrm{NaCl}$, confirming that $\mathrm{Nacl}$ contributes to the genetic traits.

$\mathrm{Nac} 1$ is involved in developmental processes because $\Delta$ nacl produces fewer and smaller conidia and more hyphal branches than wild type. The formation of conidia by A. alternata is closely regulated by the $\mathrm{G}$ protein and the cAMP-dependent protein kinase as well as the Fus3 and Slt2 MAPKs- and the calcium-mediated signaling pathways (Lin et al. 2010; Tsai et al. 2013; Tsai and Chung 2014; Wang et al. 2010; Yago et al. 2011). Other factors including the $S k n 7$ response regulator, NADPH oxidase (Nox), glutathione peroxidase (Gpx3), glutathione reductase (Glr1), thioredoxin reductase (Trr1), and nonribosomal peptide synthetase (Nps6) are also required for the formation and development of conidia in A. alternata (Chen et al. 2012; Ma et al. 2018; Yang and Chung 2013; Yang et al. 2016). The results suggest a close link between Nac1 and those regulators in terms of the formation of conidia. However, the exact interactions among these regulators leading to conidial development remain unknown at this point. Although Nac1 is
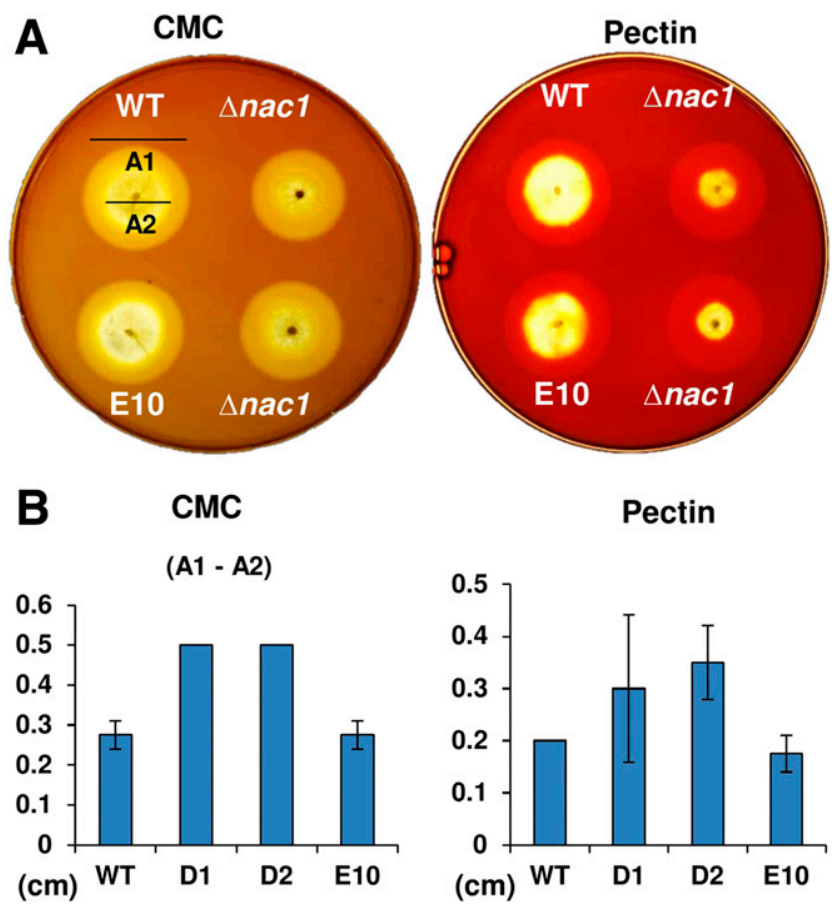

Fig. 6. The nascent polypeptide-associated complex $\alpha$ subunit (Nac1) negatively regulates cell wall-degrading enzymes. A, Agar plate assays for the production of cellulase and pectolytic enzymes by wild-type (WT), heterokaryotic (E10), and $\Delta n a c 1$ strains of Alternaria alternata. Fungal strains were cultured on a synthetic medium amended with carboxymethyl cellulose (CMC) or pectin for 3 to 5 days. Cellulase activity was determined by the formation of clear halos around colonies after staining with Gram's iodine. Pectolytic activity was determined after staining with $0.1 \%$ Congo red. B, Quantitative analysis of cell wall-degrading enzymes. Each column represents the mean value of size (diameter A1 - diameter A2) \pm the standard error from two independent experiments with at least three replicates. apparently required for growth, Nac1 negatively controls hyphal branching, confirming further the role of Nac1 in fungal development. These developmental deficiencies may be an important attribute to reduced virulence in $\Delta n a c 1$ as the mutant incites smaller lesions on unwounded leaves. However, $\Delta$ nacl produces wild-type lesions on citrus leaves that are wounded before inoculation. This is likely due to the fact that $\Delta$ nacl can successfully deploy ACT once it gets inside the host tissues.

As shown in the present study, Nac1 negatively regulates cellular resistance to high levels of glucose, mannitol (a type of sugar alcohol), and sorbitol (also sugar alcohol); however, $\Delta$ nacl is required for resistance to sucrose, $\mathrm{NaCl}$, and $\mathrm{KCl}$. Previous studies have demonstrated that A. alternata could resist high concentrations of sugars and salts, mainly via response regulators $\mathrm{Skn} 7$ and $\mathrm{Ssk} 1$, respectively. $\mathrm{Skn} 7$ is primarily responsible for resistance to sugar-induced osmotic stress via the histidine kinase (Hsk1)-mediated phosphorelay signaling pathway; however, $\mathrm{Skn} 7$ plays no role in resistance to $\mathrm{NaCl}$ and $\mathrm{KCl}$ salts (Chen et al. 2012). Comparative qRT-PCR analysis reveals that the expression of $S k n 7$ is negatively regulated by Nac1, consistent with the notion that Skn7 and Nac1 play an antagonistic role in osmotic stress induced by glucose, mannitol, or sorbitol. However, Nac1 plays an opposite role in resistance to monosaccharide (glucose) and disaccharide (sucrose), both of which are regulated by $\mathrm{Skn} 7$. Whether or how Skn7 and Nac1 can counterbalance and lead to sucrose-induced osmotic stress resistance remains unknown. It is also likely that $\mathrm{Nac} 1$ and $\mathrm{Skn} 7$ might regulate different pathways in response to a high level of sucrose. In A. alternata, cellular resistance to salts is primarily mediated by the Ssk1 and its downstream Hog1 MAPK-mediated pathway and the Ssk1-Hog1 pathway plays no role in resistance to high levels of sugars (Lin and Chung 2010; Yu et al. 2016). Although both Hog1 and Nac1 are required for salt resistance, it is not likely the interaction occurs at the transcriptional level. Deletion of Hogl leads to the decreased expression of $\mathrm{Nacl}$. In contrast, inactivation of $\mathrm{Nacl}$ increases the accumulation of the Hogl transcript (Fig. 8A). Whether or not deletion of $\mathrm{Nacl}$ will impact the posttranslational modification of Hog1 (e.g., phosphorylation) remains uncertain at this point.

In addition to osmotic stress, Nac1 plays a negative role in ROS resistance as the Nacl null mutant decreases sensitivity to $\mathrm{H}_{2} \mathrm{O}_{2}$, eosin $\mathrm{Y}$, and rose bengal (both are singlet oxygengenerating compounds), and 2-chloro-5-hydroxypyridine (a potent producer of superoxide and hydroxyl radicals). Previous studies have shown that $A$. alternata employs effective mechanisms to deal with the toxicity of $\mathrm{H}_{2} \mathrm{O}_{2}$ and ROS, and the ability to detoxify ROS is required for fungal invasion to the host plant. Through analysis of loss- and gain-of-function mutations, several key factors including Yap1, Hog1, Skn7, Nox, and Nps6 have been shown to be required for ROS resistance and full virulence on citrus (Chung 2014). Nox is responsible for low-level $\mathrm{H}_{2} \mathrm{O}_{2}$ production, which could activate the expression of Yapl, Hogl, and Skn7 (Yang and Chung 2013). Those regulators in turn activate the genes required for ROS detoxification and resistance. Nox also regulates the expression of Nacl. Nac1 apparently represses the expression of NoxA, NoxB, and NoxR. This transcriptional feedback loop could avoid excessive production of toxic ROS by NADPH oxidases. Similar feedback regulatory mechanisms of Nox by Yap1 and Hog1 have been observed previously (Yang and Chung 2013). Because deletion of Nacl increases the expression of Yapl, Hogl, and $S k n 7$, all implicated in ROS resistance, may explain why $\Delta$ nacl increases resistance to $\mathrm{H}_{2} \mathrm{O}_{2}$ and other ROS-producing compounds.

Iron plays an important role in ROS resistance because it could activate antioxidant activities. Studies have shown that 

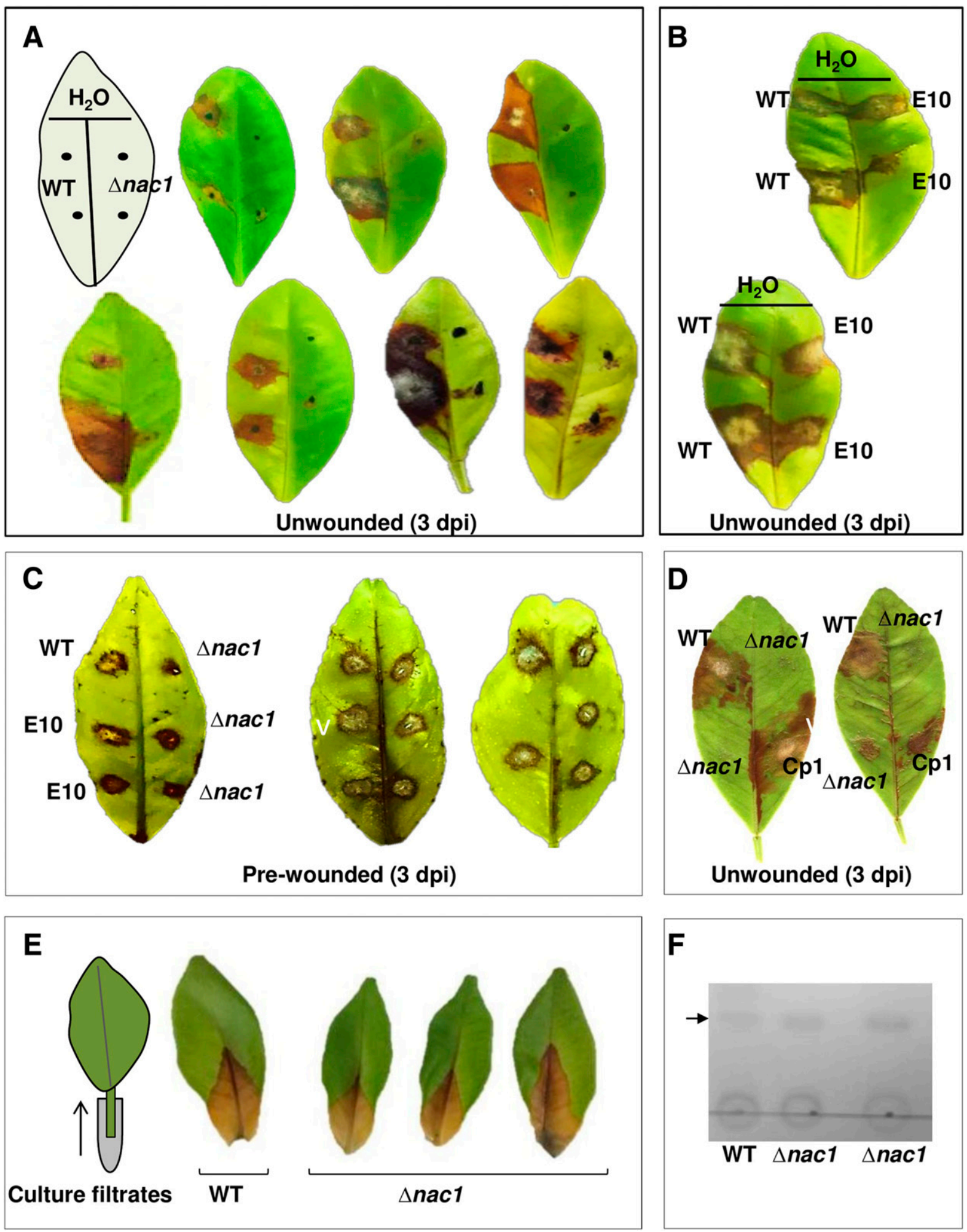

Fig. 7. Mutation of the nascent polypeptide-associated complex $\alpha$ subunit gene (Nacl) affects Alternaria alternata virulence. A, Fungal pathogenicity was assayed on detached calamondin leaves. Each spot was inoculated with $5 \mu 1$ of conidial suspensions ( $10^{4}$ conidia per milliliter) prepared from the wild type (WT) or $\Delta$ nacl mutant strains. B, Necrotic lesions on calamondin leaves inoculated with WT and the E10 heterokaryotic strain 3 days postinoculation (dpi). $\Delta$ nacl induced minor or no visible lesions $3 \mathrm{dpi}$. $\mathrm{H}_{2} \mathrm{O}$ treatment was used as a mock control. C, Development of necrotic lesions on calamondin leaves wounded before inoculation with A. alternata strains. D, The complementation strain $(\mathrm{Cp} 1)$ induced necrotic lesions similar to WT. E, A leaf necrosis assay for the toxicity of Alternaria citri toxin (ACT) by soaking the petiole of the leaf in cell-free culture filtrates in a 1.5 -ml microcentrifuge tube placed in a plastic rack for 3 days. The leaves were maintained in a moisture chamber for lesion development. ACT produced by A. alternata strains was translocated along the major vein and resulted in the development of necrotic lesions. F, Thin-layer chromatography analysis of ACT purified from culture filtrates. 
A. alternata strains lacking Yap1, Hog1, Skn7, or Nox all decrease the production of siderophores (iron chelators) and increase cellular sensitivity to ROS (Chen et al. 2014). Iron could partially restore ROS sensitivity associated with a fungal mutant lacking Yap1, Hog1, Skn7, Nox, or Nps6. The ability to acquire iron via dimethyl coprogen siderophores plays a critical role in ROS detoxification and the production of siderophores is coordinately regulated by Nox, Yap1, and Hog1 under ironlimiting conditions in A. alternata. Although $\mathrm{Skn} 7$ regulates iron uptake, $\mathrm{Skn} 7$ is not involved in siderophore production (Chen et al. 2014). In addition to antioxidant activities, Yap1, Hog1, and Skn7 play a profound role in the regulation of membrane-bound major facilitator superfamily transporters, which are also required for siderophore uptake (C.-W. Chen unpublished data) and ROS resistance (Chen et al. 2017; Lin et al. 2018).
Alternaria species produce dimethyl coprogen siderophores belonging to the hydroxamate group (Jalal and van der Helm 1989; Ohra et al. 1995; Oide et al. 2006) and siderophores are required for ROS resistance in A. alternata. Genetic evidence has shown that inactivation of Nacl results in a reduced production of siderophores; however, $\Delta$ nacl displays increased resistance to ROS, as assayed on PDA. This discrepancy is likely because $A$. alternata utilizes a reductive iron assimilation mechanism (Haas et al. 2008) rather than siderophores to acquire iron under iron-rich medium. The requirement of $\mathrm{Nac} 1$ in the production of siderophores is supported by qRT-PCR analysis, revealing that the expression of $\mathrm{Nacl}$ is inducible by iron. The expression of the SidA, Nps6, Sit1, MirC, and Atf1 genes encoding proteins involved in siderophore biosynthesis, regulation, and transportation is regulated by Nac1, further
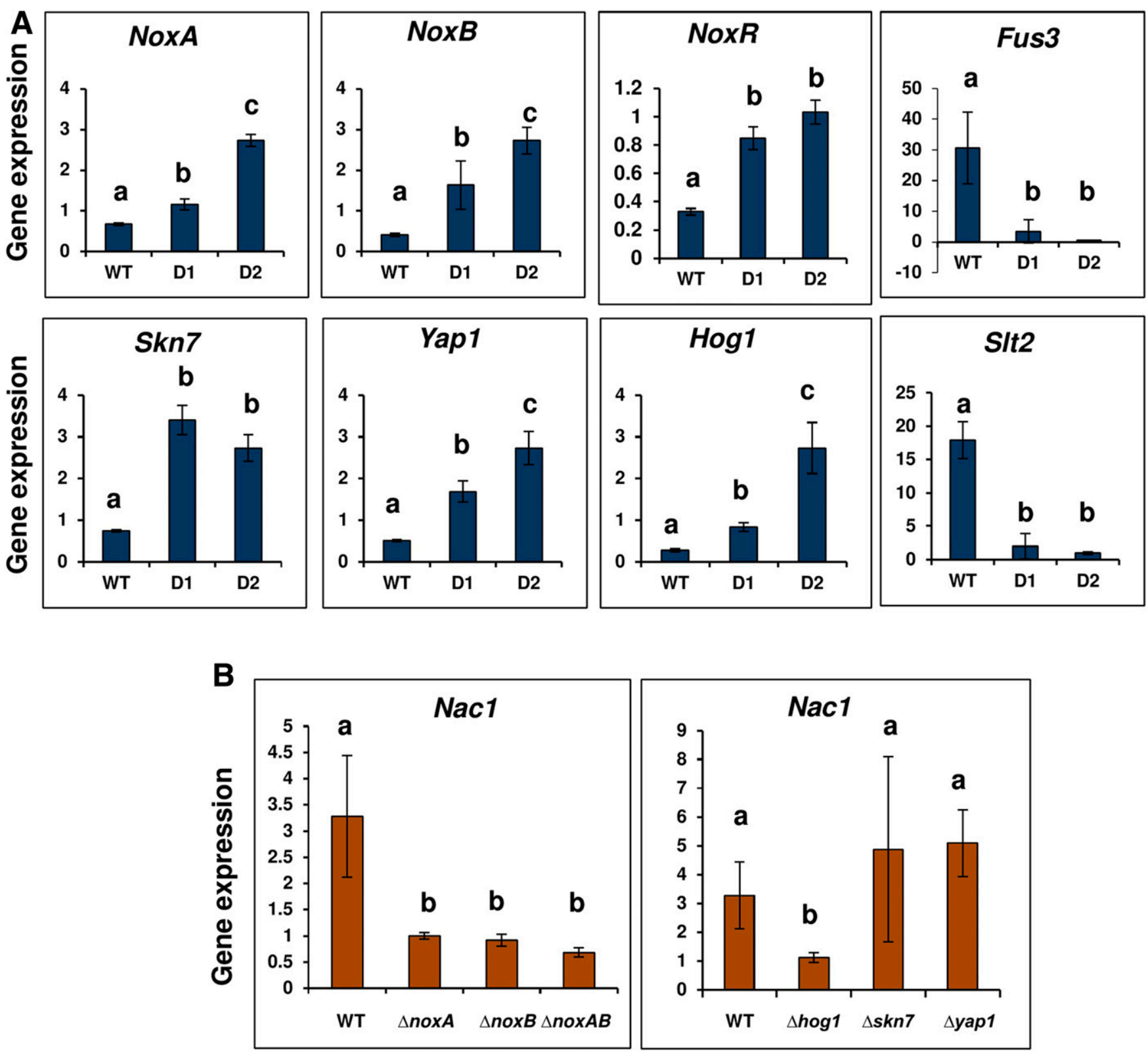

Fig. 8. Quantitative real-time PCR (qRT-PCR) analysis. A, The expression of the genes encoding NADPH oxidase subunits (NoxA, NoxB, and NoxR), response regulator Skn7, redox transcription regulator Yap1, as well as three mitogen-activated protein kinases, namely, Hog1, Fus3, and Slt2, in the wild type (WT) and two $\Delta$ nac1 mutants (D1 and D2) of Alternaria alternata. B, Accumulation of the nascent polypeptide-associated complex $\alpha$ subunit (Nac1) transcript in WT and fungal strains lacking NoxA $(\Delta$ noxA $)$, NoxB $(\Delta$ noxB $)$, NoxA NoxB $(\Delta n o x A B$ double mutant), Yap1 $(\Delta y a p 1), \operatorname{Skn} 7(\Delta s k n 7)$, or Hog1 $(\Delta h o g 1)$. The relative expression levels from three independent reactions were calculated using a comparative cycle threshold method in relation to the expression of a fungal $\beta$ tubulin-coding gene. Means indicated by the same letter are not significantly different from one another, $P<0.05$. 
confirming the requirement of Nac1 in the production and transportation of siderophores.

Virulence assays on detached calamondin leaves reveal that $\Delta n a c 1$ induces much smaller necrotic lesions on unwounded leaves than wild type but induced wild-type lesions on citrus leaves that were wounded before inoculation. $\Delta n a c l$ is apparently impaired in the penetration stage rather than the colonization stage. Because $\Delta n a c l$ induces less $\mathrm{H}_{2} \mathrm{O}_{2}$ accumulation at the sites of the inoculation compared with wild type, based on DAB staining (Supplementary Fig. S4), $\mathrm{H}_{2} \mathrm{O}_{2}$ is unlikely the main factor leading to reduced virulence of $\Delta n a c 1$. A. alternata can secret ACT to kill host cells before invasion and acquire nutrients primarily from dead tissues. Leaf bioassays also reveals that $\Delta n a c l$ produces ACT at a level comparable to that produced by wild type. Nonetheless, virulence reduction is very likely the result of a combination of multiple factors, because $\Delta n a c 1$ is defective for growth, hyphal development, stress response, and the ability to effectively acquire iron, all of which could impair fungal penetration. Studies with $S$. sclerotiorum have revealed that the Nac $\alpha$ subunit plays a negative role in the production of CWDEs and fungal virulence after characterizing a RNAimediated fungal mutant ( $\mathrm{Li}$ et al. 2015). In contrast, Nac1 is required for the production of cellulase and pectinase and full virulence of $A$. alternata.

In conclusion, A. alternata utilizes multifaceted signaling transduction pathways to perceive and respond to a broad diversity of environmental cues. Nac1 apparently facilitates the orchestration of important regulatory pathways, which could allow A. alternata to properly respond to environmental stress and enhance its ability to infect the host plant.

\section{MATERIALS AND METHODS}

Fungal strains and culture conditions.

A wild-type strain of A. alternata (Fr.) Keissler was isolated from a diseased leaf of Minneola (Citrus paradisi Macfad. $\times$ C. reticulata Blanco) tangelo (Lin et al. 2009) and was used for genetic manipulation in this study. Fungal strains lacking a Yap1 transcription activator, a NADPH oxidase (NoxA or NoxB single deletion and NoxA NoxB double deletion), a Skn7 response regulator, or a Hog1, Fus3, or Slt2 MAPK were created in previous studies (Lin and Chung 2010; Lin et al. 2009, 2010; Yago et al. 2011; Yang and Chung 2012, 2013; Yang et al. 2016). Fungi were grown on PDA under constant illumination for 5 to 7 days for conidial formation. The concentration of conidia was determined with the aid of a hemacytometer. Conidial germination was conducted by placing conidial suspensions on a glass slide, incubating them in a moist chamber for 6 $\mathrm{h}$, and examining them with a Leitz Laborlux phase contrast microscope (Leitz Wetzlar). For DNA isolation, fungal strains were grown on a layer of cellophane overlaid on PDA (Difco). Fungi were cultured in a Richard's solution (Kohmoto et al. 1993) for at least 3 weeks when the production of a hostselective ACT was desired. Cell-free culture filtrates used for a leaf bioassay were prepared by passing through a $0.45 \mu \mathrm{m}$ polyethersulfone (PES) membrane (Thermo Fisher Scientific). The regeneration medium (RMM), containing $1 \mathrm{M}$ sucrose as an osmotic reagent (Chung et al. 2002), was used to recover fungi after transformation. Sensitivity assays were conducted on PDA or MM (Chen et al. 2013) by transferring fungal mycelia as a toothpick point-inoculation to medium containing a test compound at an appropriate concentration, and plates were incubated under constant fluorescent light. Percentage of growth inhibition was calculated by dividing the relative difference of growth by the wild-type growth and multiplying by 100 .

\section{Generation of split-marker fragments and} targeted gene disruption.

The A. alternata Nacl fragment (formerly Ape1, accession number Kx134679) (Lin et al. 2017) was amplified by PCR with the primers from genomic DNA prepared from wild type. Fungal DNA was purified using a DNeasy plant mini kit (Qiagen). Nacl was mutated by integrating a bacterial phosphotransferase B gene $(H Y G)$ cassette under control of the Aspergillus nidulans $\operatorname{Trp} C$ gene promoter and terminator conferring resistance to hygromycin in the genome of A. alternata, using a split-marker approach (Chung and Lee 2014). A $5^{\prime} \mathrm{Nac} 1:: 5^{\prime} \mathrm{HYg}$ fragment $(2+1.2 \mathrm{~kb})$ was generated by tworound PCR with primers ALAF1, ALA13R, M13R, and Hyg3 (Supplementary Fig. S3). A $3^{\prime} \mathrm{Nac} 1:: 3^{\prime} \mathrm{hYG}$ fusion fragment $(1.8+2.1 \mathrm{~kb})$ was generated by fusion PCR with the primers Hyg4, M13F, ALA13F, and ALAR1. The primers ALA13R and ALA13F contain the oligonucleotides completely complementary to the sequence of the primers M13R and M13F, respectively. PCR fragments (10 $\mu \mathrm{l}$ each) were mixed and were transformed into fungal protoplasts prepared from the wild-type strain of $A$. alternata, using polyethylene glycol and $\mathrm{CaCl}_{2}$, as previously described (Chung and Lee 2014; Chung et al. 2002). Fungal protoplasts were generated by treating hyphae with a combination of CWDEs, including $\beta$-glucuronidase (type $\mathrm{H} 2$; Sigma-Aldrich), lysing enzyme (Sigma-Aldrich), and driselase, as described (Chung et al. 2002). Fungal transformants appearing on RMM amended with $250 \mu \mathrm{g}$ of hygromycin per milliliter (Roche Applied Science) were individually transferred to PDA. The integration of $H Y G$ within Nacl was examined by Southern blot analysis, using a Nacl-specific probe and a $H Y G$ gene-specific probe. Fungal genomic DNA was cleaved with $X b a \mathrm{I}$, was electrophoresed in a $0.7 \%$ agarose gel, was blotted to a nylon membrane, and was hybridized with a gene-specific probe. DNA probe was amplified and labeled by PCR by incorporating digoxigenin (DIG-11-dUTP) into the probe with the Nacl- or $H Y G$-specific primers. The probe was detected by immunological assays using CSPD (disodium 3-[4methoxyspiro \{1,2-dioxetane-3,2'-[5'-chloro]tricyclo [3.3.1.1] decan -4-yl]phenyl phosphate) as a chemofluorescent substrate (Roche Applied Science) for alkaline phosphatase, according to manufacturer recommendations. Oligonucleotide primers and their sequences used in this study are listed in Supplementary Table S1.

\section{Genetic complementation.}

A functional copy of $\mathrm{Nacl}$ under control of its endogenous promoter $(1 \mathrm{~kb})$ was amplified by a high-fidelity DNA polymerase with Nacl-specific primers NacI_BamHI_com_nF and NacI_ApaI_com_nR (Supplementary Table S1) and was cloned into pCB1532 carrying the Magnaporthe grisea acetolactate synthase gene cassette that confers sulfonylurea resistance (Sweigard et al. 1997), to yield the complementation construct pNac1::pCB1532. Endonuclease recognition sites BamHI and ApaI were incorporated into the primers to facilitate cloning. The construct was transformed into protoplasts prepared from a $\Delta$ nacl mutant (D1). Transformants were regenerated on RMM containing $5 \mu \mathrm{g}$ of sulfonylurea per milliliter (chlorimuron ethyl; Chem Service). Transformants were picked and were examined for the restoration of radial growth on PDA and for PCR with two Nacl-specific primers.

\section{Assays for siderophore production.}

The production of extracellular siderophores was tested using a CAS-containing agar plate assay (Schwyn and Neilands 1987). CAS medium amended with HDTMA and $\mathrm{FeCl}_{3}$ was prepared as previously described (Chen et al. 2013). The production of siderophores by fungi resulted in the formation of 
orange halos around fungal colonies grown on CAS plates. The areas of yellow halos (minus fungal colony) produced by A. alternata strains were quantified using an Image $\mathrm{J}$ program. Siderophores were extracted from fungal cultural filtrates, grown in liquid MM for 5 to 7 days, by passing through an Amberlite XAD-16 column (Oide et al. 2006). Fungal mycelium was separated from culture filtrates by vacuum filtration through a pre-weighed filter paper. The filter papers were dried and were weighed again to estimate the overall fungal mass. Siderophores were eluted with methanol, were dried at room temperature (approximately $25^{\circ} \mathrm{C}$ ), and were re-dissolved in an appropriate volume of methanol, based on fungal mass. Siderophores were separated in a 60 F254 silica gel plate using methanol/acetic acid/ $\mathrm{H}_{2} \mathrm{O}(4: 1: 5, \mathrm{vol} / \mathrm{vol} / \mathrm{vol})$ as a mobile phase.

\section{Gene expression.}

Fungal RNA was purified using TRI reagent (SigmaAldrich), treated with RQ1 RNase-free DNase (Promega), and was used to synthesize cDNA using a One Step iScript cDNA synthesis kit (Bio-Rad) and an oligo-dT primer. qRTPCR reactions were prepared using iQ SYBR green supermix (Bio-Rad) and were conducted in a CFX Connect model of real time PCR detection system (Bio-Rad). The cycling profile for amplification was performed as follows: $95^{\circ} \mathrm{C}$ for $3 \mathrm{~min}$, followed by 40 cycles of $95^{\circ} \mathrm{C}$ for $10 \mathrm{~s}, 60^{\circ} \mathrm{C}$ for $30 \mathrm{~s}$ and one final step of $95^{\circ} \mathrm{C}$ for $10 \mathrm{~s}$. Amplification of specific genes was confirmed by melting curve; amplification of the A. alternata $\beta$-tubulin gene was used as an internal control to normalize for variation among samples. The relative expression levels were calculated using a comparative cycle threshold method (Schefe et al. 2006).

\section{Assays for fungal virulence and toxin production.}

Fungal virulence was performed on detached calamondin (Citrus mitis Blanco) leaves as previously described (Yang et al. 2016). Citrus leaves were inoculated by placing $5 \mu \mathrm{l}$ of conidial suspensions $\left(10^{4}\right.$ conidia per milliliter) or fungal mycelium (toothpick inoculation) on each spot. Virulence was also assayed on citrus leaves that were wounded with a fine needle prior to inoculation. The mock controls were treated with water only. The treated leaves were placed in a plastic box for lesion formation. Each fungal strain was tested on at least five leaves and experiments were repeated three times. A leaf necrosis assay for the toxicity of ACT was performed by soaking the petiole of the leaf in cell-free culture filtrates in a $1.5-\mathrm{ml}$ microcentrifuge tube, as described by Kohmoto et al. (1993). ACT was also extracted from culture filtrates with Amberlite XAD-2 and ethyl acetate and were analyzed by TLC. Accumulation of $\mathrm{H}_{2} \mathrm{O}_{2}$ in citrus leaves was determined using DAB as a chromogenic substrate (Orozco-Cárdenas and Ryan 1999). $\mathrm{H}_{2} \mathrm{O}_{2}$ was identified by the accumulation of a brownish color due to polymerization of DAB.

\section{Assays for CWDEs.}

Extracellular activities of CWDEs were evaluated by culturing fungal strains on a synthetic medium $(0.2 \mathrm{~g}$ of $\mathrm{NaNO}_{3}, 0.1 \mathrm{~g}$ of $\mathrm{K}_{2} \mathrm{HPO}_{4}, 0.05 \mathrm{~g}$ of $\mathrm{MgSO}_{4} \cdot 7 \mathrm{H}_{2} \mathrm{O}, 0.05 \mathrm{~g}$ of $\mathrm{KCl}, 0.02 \mathrm{~g}$ of peptone, and $1.7 \mathrm{~g}$ of agar per $100 \mathrm{ml}$ ) amended with $0.2 \mathrm{~g}$ of carboxymethyl cellulose or pectin for 3 to 5 days (Johnsen and Krause 2014). Cellulase activity was assessed after staining with Gram's iodine ( $2 \mathrm{~g}$ of KI and $1 \mathrm{~g}$ of iodine per $300 \mathrm{ml}$ ). Pectolytic activity was determined after staining with $0.1 \%$ Congo red and de-staining by $1 \mathrm{M}$ $\mathrm{NaCl}$, as described by Kasana et al. (2008). The CWDE activity was determined by the production of halos around the colonies.

\section{Statistical analysis.}

All experiments were conducted three times with at least three replicates. The significance of treatments was determined by one-way analysis of variance and treatment separated by Tukey's honest significant difference post hoc test $(P<0.05)$.

\section{ACKNOWLEDGMENTS}

The authors thank H. C. Lin for his able assistance.

\section{LITERATURE CITED}

Adams, T. H., Wieser, J. K., and Yu, J. H. 1998. Asexual sporulation in Aspergillus nidulans. Microbiol. Mol. Biol. Rev. 62:35-54.

Bloss, T. A., Witze, E. S., and Rothman, J. H. 2003. Suppression of CED-3independent apoptosis by mitochondrial $\beta \mathrm{NAC}$ in Caenorhabditis elegans. Nature 424:1066-1071.

Chen, L.-H., Lin, C.-H., and Chung, K.-R. 2012. Roles for SKN7 response regulator in stress resistance, conidiation and virulence in the citrus pathogen Alternaria alternata. Fungal Genet. Biol. 49:802-813.

Chen, L.-H., Lin, C.-H., and Chung, K.-R. 2013. A nonribosomal peptide synthetase mediates siderophore production and virulence in the citrus fungal pathogen Alternaria alternata. Mol. Plant Pathol. 14:497-505.

Chen, L.-H., Tsai, H.-C., Yu, P. L., and Chung, K. R. 2017. A major facilitator superfamily transporter-mediated resistance to oxidative stress and fungicides requires Yap1, Skn7, and MAP kinases in the citrus fungal pathogen Alternaria alternata. PLoS One 12:e0169103.

Chen, L.-H., Yang, S. L., and Chung, K.-R. 2014. Resistance to oxidative stress via regulating siderophore-mediated iron acquisition by the citrus fungal pathogen Alternaria alternata. Microbiology 160:970-979.

Chung, K. -R. 2012. Stress response and pathogenicity of the necrotrophic fungal pathogen Alternaria alternata. Scientifica 2012:635431.

Chung, K.-R. 2014. Reactive oxygen species in the citrus fungal pathogen Alternaria alternata: The role of NADPH-dependent oxidase. Physiol. Mol. Plant Pathol. 88:10-17.

Chung, K.-R., and Lee, M.-H. 2014. Split marker-mediated transformation and targeted gene disruption in filamentous fungi. Pages 175-180 in: Genetic Transformation System in Fungi. van den Berg, M., and Maruthachalam, K., eds. Vol. 2. Springer International Publishing, Switzerland.

Chung, K.-R., Shilts, T., Li, W., and Timmer, L. W. 2002. Engineering a genetic transformation system for Colletotrichum acutatum, the causal fungus of lime anthracnose and postbloom fruit drop of citrus. FEMS Microbiol. Lett. 213:33-39.

Creagh, E. M., Brumatti, G., Sheridan, C., Duriez, P. J., Taylor, R. C., Cullen, S. P., Adrain, C., and Martin, S. J. 2009. Bicaudal is a conserved substrate for Drosophila and mammalian caspases and is essential for cell survival. PLoS One 4:e5055.

del Alamo, M., Hogan, D. J., Pechmann, S., Albanese, V., Brown, P. O., and Frydman, J. 2011. Defining the specificity of cotranslationally acting chaperones by systematic analysis of mRNAs associated with ribosomenascent chain complexes. PLoS Biol. 9:e1001100.

Deng, J. M., and Behringer, R. R. 1995. An insertional mutation in the BTF3 transcription factor gene leads to an early postimplantation lethality in mice. Transgenic Res. 4:264-269.

Espeso, E. A., Tilburn, J., Sánchez-Pulido, L., Brown, C. V., Valencia, A., Arst, H. N., Jr., and Peñalva, M. Á. 1997. Specific DNA recognition by the Aspergillus nidulans three zinc finger transcription factor PacC. J. Mol. Biol. 274:466-480.

Franke, J., Reimann, B., Hartmann, E., Köhlerl, M., and Wiedmann, B. 2001. Evidence for a nuclear passage of nascent polypeptide-associated complex subunits in yeast. J. Cell Sci. 114:2641-2648.

George, R., Walsh, P., Beddoe, T., and Lithgow, T. 2002. The nascent polypeptide-associated complex (NAC) promotes interaction of ribosomes with the mitochondrial surface in vivo. FEBS Lett. 516:213-216.

Guo, B., Huang, J., Wu, W., Feng, D., Wang, X., Chen, Y., and Zhang, H. 2014. The nascent polypeptide-associated complex is essential for autophagic flux. Autophagy 10:1738-1748.

Haas, H., Eisendle, M., and Turgeon, B. G. 2008. Siderophores in fungal physiology and virulence. Annu. Rev. Phytopathol. 46:149-187.

Hartl, F. U., and Hayer-Hartl, M. 2002. Molecular chaperones in the cytosol: From nascent chain to folded protein. Science 295:1852-1858.

Hotokezaka, Y., van Leyen, K., Lo, E. H., Beatrix, B., Katayama, I., Jin, G., and Nakamura, T. 2009. alphaNAC depletion as an initiator of ER stressinduced apoptosis in hypoxia. Cell Death Differ. 16:1505-1514. 
Jalal, M. A. F., and van der Helm, D. 1989. Siderophores of highly phytopathogenic Alternaria longipes: Structures of hydroxycoprogens. Biol. Met. 2:11-17.

Johnsen, H. R., and Krause, K. 2014. Cellulase activity screening using pure carboxymethylcellulose: Application to soluble cellulolytic samples and to plant tissue prints. Int. J. Mol. Sci. 15:830-838.

Kasana, R. C., Salwan, R., Dhar, H., Dutt, S., and Gulati, A. 2008. A rapid and easy method for the detection of microbial cellulases on agar plates using gram's iodine. Curr. Microbiol. 57:503-507.

Kim, S. H., Shim, K. S., and Lubec, G. 2002. Human brain nascent polypeptide-associated complex alpha subunit is decreased in patients with Alzheimer' s disease and Down syndrome. J. Investig. Med. 50:293-301.

Kohmoto, K., Itoh, Y., Shimomura, N., Kondoh, Y., Otani, H., Nishimura, S., and Nakatsuka, S. 1993. Isolation and biological activities of two host-specific toxins from tangerine pathotype of Alternaria alternata. Phytopathology 83:495-502.

Kroes, R. A., Jastrow, A., McLone, M. G., Yamamoto, H., Colley, P., Kersey, D. S., Yong, V. W., Mkrdichian, E., Cerullo, L., Leestma, J., and Moskal, J. R. 2000. The identification of novel therapeutic targets for the treatment of malignant brain tumors. Cancer Lett. 156:191-198.

Li, X., Guo, M., Xu, D., Chen, F., Zhang, H., Pan, Y., Li, M., and Gao, Z. 2015. The nascent-polypeptide-associated complex alpha subunit regulates the polygalacturonases expression negatively and influences the pathogenicity of Sclerotinia sclerotiorum. Mycologia 107: 1130-1137.

Lin, C.-H., and Chung, K.-R. 2010. Specialized and shared functions of the histidine kinase- and HOG1 MAP kinase-mediated signaling pathways in Alternaria alternata, a filamentous fungal pathogen of citrus. Fungal Genet. Biol. 47:818-827.

Lin, C.-H., Yang, S. L., and Chung, K.-R. 2009. The YAP1 homologmediated oxidative stress tolerance is crucial for pathogenicity of the necrotrophic fungus Alternaria alternata in citrus. Mol. Plant-Microbe Interact 22:942-952.

Lin, C.-H., Yang, S. L., Wang, N.-Y., and Chung, K.-R. 2010. The FUS3 MAPK signaling pathway of the citrus pathogen Alternaria alternata functions independently or cooperatively with the fungal redoxresponsive AP1 regulator for diverse developmental, physiological and pathogenic processes. Fungal Genet. Biol. 47:381-391.

Lin, H.-C., Yu, P.-L., Chen, L.-H., Tsai, H.-C., and Chung, K.-R. 2018. A major facilitator superfamily transporter regulated by the stressresponsive transcription factor Yap1 is required for resistance to fungicides, xenobiotics, and oxidants and full virulence in Alternaria alternata. Front. Microbiol. 9:2229.

Lin, T.-C., Hsieh, T.-Y., Lin, C.-L., Chung, W.-C., Chung, K.-R., and Huang, J.-W. 2017. Pathogenic fungal protein-induced resistance and its effects on vegetable diseases. J. Agric. Sci. 155:1069-1081.

Linden, H., and Macino, G. 1997. White collar-2, a partner in blue light signal transduction, controlling expression of light-regulated genes in Neurospora crassa. EMBO J. 16:98-107.

Ma, H., Wang, M., Gai, Y., Fu, H., Zhang, B., Ruan, R., Chung, K.-R., and Li, H. 2018. Thioredoxin and glutaredoxin systems required for oxidative stress resistance, fungicide sensitivity and virulence of Alternaria alternata. Appl. Environ. Microbiol. 4:e00086-18.

Markesich, D. C., Gajewski, K. M., Nazimiec, M. E., and Beckingham, K. 2000. Bicaudal encodes the Drosophila beta NAC homolog, a component of the ribosomal translational machinery. Development 127:559-572.

Marzluf, G. A. 1997. Genetic regulation of nitrogen metabolism in the fungi. Microbiol. Mol. Biol. Rev. 61:17-32.

Meng, F., Xiao, Y., Guo, L., Zeng, H., Yang, X., and Qiu, D. 2018. A DREPP protein interacted with PeaT1 from Alternaria tenuissima and is involved in elicitor-induced disease resistance in Nicotiana plants. J. Plant Res. 131:827-837.

Ohra, J., Morita, K., Tsujino, Y., Fujimori, T., Goering, M., Evans, S., and Zorner, P. 1995. Production of two phytotoxic metabolites by the fungus Alternaria cassia. Biosci. Biotechnol. Biochem. 59:1782-1783.

Oide, S., Moeder, W., Krasnoff, S., Gibson, D., Haas, H., Yoshioka, K., and Turgeon, B. G. 2006. NPS6, encoding a nonribosomal peptide synthetase involved in siderophore-mediated iron metabolism, is a conserved virulence determinant of plant pathogenic ascomycetes. Plant Cell 18: 2836-2853.

Orozco-Cárdenas, M., and Ryan, C. A. 1999. Hydrogen peroxide is generated systemically in plant leaves by wounding and systemin via the octadecanoid pathway. Proc. Natl. Acad. Sci. U.S.A. 96:6553-6557.

Ott, A.-K., Locher, L., Koch, M., and Deuerling, E. 2015. Functional dissection of the nascent polypeptide-associated complex in Saccharomyces cerevisiae. PLoS One 10:e143457.
Pech, M., Spreter, T., Beckmann, R., and Beatrix, B. 2010. Dual binding mode of the nascent polypeptide-associated complex reveals a novel universal adapter site on the ribosome. J. Biol. Chem. 285 19679-19687.

Preissler, S., and Deuerling, E. 2012. Ribosome-associated chaperones as key players in proteostasis. Trends Biochem. Sci. 37:274-283.

Rangan, V. S., Oskouian, B., and Smith, S. 1996. Identification of an inverted CCAAT box motif in the fatty-acid synthase gene as an essential element for mediation of transcriptional regulation by cAMP. J. Biol. Chem. 271:2307-2312.

Reimann, B., Bradsher, J., Franke, J., Hartmann, E., Wiedmann, M., Prehn, S., and Wiedmann, B. 1999. Initial characterization of the nascent polypeptide-associated complex in yeast. Yeast 15:397-407.

Rospert, S., Dubaquié, Y., and Gautschi, M. 2002. Nascent-polypeptideassociated complex. Cell. Mol. Life Sci. 59:1632-1639.

Schefe, J. H., Lehmann, K. E., Buschmann, I. R., Unger, T., and FunkeKaiser, H. 2006. Quantitative real-time RT-PCR data analysis: Current concepts and the novel "gene expression's CT difference" formula. J. Mol. Med. (Berl.) 84:901-910.

Scheuring, U. J., Corbeil, J., Mosier, D. E., and Theofilopoulos, A. N. 1998. Early modification of host cell gene expression induced by HIV-1. AIDS 12:563-570.

Schwyn, B., and Neilands, J. B. 1987. Universal chemical assay for the detection and determination of siderophores. Anal. Biochem. 160:47-56.

Shi, F., Dong, Y., Zhang, Y., Yang, X., and Qiu, D. 2017. Overexpression of the PeaT1 elicitor gene from Alternaria tenuissima improves drought tolerance in rice plants via interaction with a Myo-inositol oxygenase. Front. Plant Sci. 8:970.

Sweigard, J. A., Chumley, F. C., Carroll, A. M., Farrall, L., and Valent, B. 1997. A series of vectors for fungal transformation. Fungal Genet Newsl. 44:52-53.

Thomma, B. P. H. J. 2003. Alternaria spp.: From general saprophyte to specific parasite. Mol. Plant Pathol. 4:225-236.

Tsai, H.-C., and Chung, K.-R. 2014. Calcineurin phosphatase and phospholipase $\mathrm{C}$ are required for developmental and pathological functions in the citrus fungal pathogen Alternaria alternata. Microbiology 160:1453-1465.

Tsai, H.-C., Yang, S. L., and Chung, K.-R. 2013. Cyclic AMP-dependent protein kinase A negatively regulates conidia formation by the tangerine pathotype of Alternaria alternata. World J. Microbiol. Biotechnol. 29: 289-300.

Wang, N.-Y., Lin, C.-H., and Chung, K.-R. 2010. A Ga subunit gene is essential for conidiation and potassium efflux but dispensable for pathogenicity of Alternaria alternata on citrus. Curr. Genet. 56:43-51.

Wang, S., Sakai, H., and Wiedmann, M. 1995. NAC covers ribosomeassociated nascent chains thereby forming a protective environment for regions of nascent chains just emerging from the peptidyl transferase center. J. Cell Biol. 130:519-528.

Whitby, M. C., and Dixon, J. 2001. Fission yeast nascent polypeptideassociated complex binds to four-way DNA junctions. J. Mol. Biol. 306: 703-716.

Wiedmann, B., and Prehn, S. 1999. The nascent polypeptide-associated complex (NAC) of yeast functions in the targeting process of ribosomes to the ER membrane. FEBS Lett. 458:51-54.

Yago, J. I., Lin, C.-H., and Chung, K.-R. 2011. The SLT2 mitogen-activated protein kinase-mediated signaling pathway governs conidiation, morphogenesis, fungal virulence, and production of toxin and melanin in the tangerine pathotype of Alternaria alternata. Mol. Plant Pathol. 12: 653-665.

Yang, S. L., and Chung, K.-R. 2012. The NADPH oxidase-mediated production of hydrogen peroxide $\left(\mathrm{H}_{2} \mathrm{O}_{2}\right)$ and resistance to oxidative stress in the necrotrophic pathogen Alternaria alternata of citrus. Mol. Plant Pathol. 13:900-914.

Yang, S. L., and Chung, K.-R. 2013. Similar and distinct roles of NADPH oxidase components in the tangerine pathotype of Alternaria alternata. Mol. Plant Pathol. 14:543-556.

Yang, S. L., Yu, P.-L., and Chung, K.-R. 2016. The glutathione peroxidasemediated reactive oxygen species resistance, fungicide sensitivity and cell wall construction in the citrus fungal pathogen Alternaria alternata. Environ. Microbiol. 18:923-935.

Yu, P.-L., Chen, L.-H., and Chung, K.-R. 2016. How the pathogenic fungus Alternaria alternata copes with stress via the response regulators SSK1 and SHO1. PLoS One 11:e0149153.

Zhang, W., Yang, X., Qiu, D., Guo, L., Zeng, H., Mao, J., and Gao, Q. 2011. PeaT1-induced systemic acquired resistance in tobacco follows salicylic acid-dependent pathway. Mol. Biol. Rep. 38:2549-2556. 